\title{
Effectiveness of Acupuncture and Acupuncture-like Transcutaneous Electrical Nerve Stimulation (ACUTENS) in treating primary dysmenorrhea- A randomised controlled trial (RCT)
}

\author{
*Sokunbi OG (PhD), Rosemary, L (MSc), Robinson, P (BSc) \\ ${ }^{I}$ Senior Lecturer, Department of Medical Rehabilitation, College of Medical Sciences University of Maiduguri \\ ${ }^{2,3}$ Rotherham Community Hospital, UK
}

\begin{abstract}
:
Objectives: the purpose of this study was to compare the effectiveness of acupuncture and study to acupuncture-like transcutaneous electrical nerve stimulation in treating primary dysmenorrhea

Methods: Subjects: Eligible women were aged 11-30 years with a diagnosis of primary dysmenorrhea. Twelve subjects each were randomized to control, acupuncture and ACUTENS groups. All subjects completed two pain questionnaires (Pain Rating Index (PRI) of McGill Pain Questionnaire and Visual Analogue Scale) before treatment; immediately post treatment; 30, 60, 120 post-treatment and the next morning upon awakening. The data were treated as means and standard deviation analyzed with one way analysis of variance and Bonferroni post hoc analysis test.
\end{abstract}

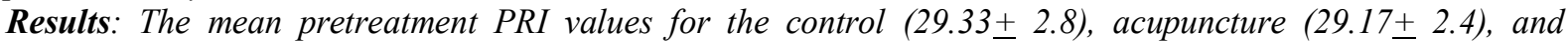
ACUTENS $(29.28 \pm 2.6)$ groups did not show statistical significant difference $(F=.016, P=0.985)$. However, $a$ decline in the mean PRI values for the acupuncture (14.42 2.49$)$ and ACUTENS $(13.83 \pm 3.13)$ groups were recorded immediately post treatment which were also statistically significant $(F=87.30, P=0.00)$. The decline in the PRI and VAS scores recorded immediately post treatments were maintained at 30mins, 60mins $120 \mathrm{~min}$ post treatment and the next day in the acupuncture and ACUTENS groups.

Similarly, the mean VAS values immediately post treatment for control $(4.75 \pm 1.76)$, acupuncture $(1.25 \pm 0.62)$ and ACUTENS $(1.33 \pm 0.65)$ showed statistical significant difference $(F=35.59, P=0.00)$. Post hoc analysis of the difference in PRI and VAS scores, between Acupuncture and ACUTENS groups did not show statistically significant difference at any point of data collection $(P>0.05)$. The overall percentage improvements in PRI scores were $10.41 \%, 50.23 \%$ and $52.26 \%$ for the control, acupuncture and ACUTENS groups respectively. Similarly, the overall improvement in VAS scores were $8.89 \%, 74.58 \%$ and $75.56 \%$ for the control, acupuncture and ACUTENS groups respectively.

Conclusion: The outcome of this study showed that acupuncture and low frequency and high intensity TENS/ acupuncture like TENS treatment could be effective in treating pain due to primary dysmenorhea. Findings from this study also showed that pain relief was approximately the same for the acupuncture and the ACUTENS groups over time.

Key words: Acupuncture, Acupuncture-like Transcutaneous Electrical Nerve stimulation, Dysmenorrhea, Pain, Physiotherapy

\section{Introduction}

Primary dysmenorhea is defined as pain during menstruation that is, not the result of an identifiable pelvic pathological condition; that is, the woman has had a normal pelvic examination. Secondary dysmenorrhea is the result of an identifiable pelvic pathological condition ${ }^{1}$. The causes of primary dysmenorrhea have not been defined specifically, but many possible causes such as psychologic and endocrine factors and cervical obstruction stenosis have been presented ${ }^{1}$ but no conclusive studies have shown these factors as the cause ${ }^{2}$. Studies have indicated an increase in intrauterine prostaglandin production and a decrease in ovarian steroid hormone as causes of primary dysmenorrhea ${ }^{1,2}$. These changes result in an elevated intrauterine pressure, which subsequently results in a decreased uterine blood flow, ischemia, and pain ${ }^{1,2}$ The prostaglandins that are released, mainlyPGF2a and PGE2, also sensitize the nerves in the uterus to the action of chemical and physical stimuli ${ }^{1}$.Dysmenorhea is a very common problem among young women, and can occur in up to $50 \%$ of menstruating women ${ }^{1}$. Symptoms include pain over the low abdomen, possibly radiating to the anterior thigh; low back pain; nausea; diarrhea; headache; fatigue; and sometimes syncope and collapse $\mathrm{e}^{1,2,3}$. These symptoms can begin a few hours or days before menstruation and last up to two or three days ${ }^{1,2,3}$. There is limited research reporting on the affect of symptoms on women's daily living activities, however studies suggest severe menstrual pain is associated with absence from school or work and restricts other activities of daily life, ${ }^{2,3}$. 
There are three approaches to the management of primary dysmenorrhea: pharmacological, nonpharmacological and surgical. Pharmacological treatment include administration of analgesics, non steroidal anti inflammatory drugs (NSAIDs) and oral contraceptives ${ }^{4,5,6}$. Evidence of efficacy supports use of pharmacological agents ${ }^{4,5,6}$, however pain relief may be inadequate for some women, or side effects may not be well tolerated ${ }^{6}$. There are also reports on the use noninvasive methods such as heat packs, biofeedback, relaxation techniques, acupressure and acupuncture to alleviate their dysmenorrhea, although precise figures describing the frequency of their use are not available ${ }^{7}$. Data are limited on patterns of use, but utilization data from the UK indicate acupuncture is used to assist with the management of gynecological or obstetric conditions by $8 \%$ of women ${ }^{7}$. Traditional acupuncture has been documented as successful in the treatment of dysmenorrhea by Macpherson et al ${ }^{7}$ and Proctor et al ${ }^{8}$. In a similar vein, one trial and two systematic reviews suggests acupuncture may reduce dysmenorrhea ${ }^{9,10}$. However, the poor methodological quality of most trials suggests a need for further rigorous research ${ }^{9,10}$.

There had also report on the preference of use of transcutaneous electrical nerve stimulation over traditional acupuncture points to achieve reduction of pain due to dysmenorhea ${ }^{11}$. Conventional TENS and "acupuncture-like" TENS are used most frequently for treatment. Conventional TENS requires a high pulse rate with a low intensity, whereas acupuncture-like TENS uses a low pulse rate with a high intensity, often as high as the patient can tolerate. Kroth studied relief of dysmenorrhea with acupuncture-like TENS in 10 women and saw significant pain relief, but no control group was used ${ }^{12 .}$ Neighbors et al studied 20 women, 10 receiving treatment of dysmenorrhea with acupuncture-like TENS and 10

receiving a placebo pill. Their results supported the hypothesis that a single treatment with acupuncture-like TENS would significantly decrease the pain of primary dysmenorrhea ${ }^{11 .}$ The aim of the present study was to compare the effectiveness of acupuncture and ACUTENS in the treatment primary dysmenorhea.

\section{Subjects}

\section{Methods}

Women were recruited from the community through their general practitioner (GP). Posters were also displayed in conspicuous areas of different GP clinics in Rotherham Community Health and Care, UK.

\section{Ethical consideration}

The Ethics Committee of the Rotherham Community Health and care, UK approved the research, and all women gave informed written consent.

\section{Inclusion criteria}

The entry criteria adopted for this trial were based on the inclusion criteria used in the Cochrane systematic review ${ }^{10}$. Women eligible to join the trial were aged 14-30 years with a diagnosis of primary dysmenorrhea. Our criteria for establishing a diagnosis of primary dysmenorrhea was based on self-reported severe incapacitating pain for at least one day of menses in two menstrual cycles classified by a pre-defined pain score of $\geq 6 / 10$ on the short form of the McGill questionnaire and a visual analog scale ${ }^{13,14}$, and pain that did not respond well to analgesics.

\section{Exclusion criteria}

Women were not eligible if they had secondary dysmenorrhea (defined as identifiable pelvic pathology), or dysmenorrhea associated with an intrauterine device. To exclude a diagnosis of secondary dysmenorhea, these women all reported having a pelvic examination within the previous two years that revealed no pathological problems or secondary dysmenorrhea. Subjects were asked to consult and clarify with their GP to confirm a diagnosis of primary dysmenorrhea prior to participation.

\section{Randomization}

A computer-generated randomization schedule was created. The randomization schedule was generated by an independent statistician. Twelve subjects each were randomized to one of the two study groups (Acupuncture and ACUTENS) and the Control (no treatment) group.

\section{Outcome measures}

Before beginning treatment, subjects completed a menstrual history questionnaire, a consent form, (PRI) of the McGill Pain Questionnaire and a visual analog scale (VAS). The PRI and VAS were used to establish a baseline level of pain. The PRI gives the subject a choice of words describing her pain at that moment. The words are assigned a rank value that allows a pain rating index (PRI) to be calculated. The VAS is composed of a 10-cm horizontal line with the words "no pain" on the left and "pain as bad as it could be" on the right. The subject is instructed to make a mark on the line at a point that represents her pain level at that time. 
These pain scales were completed immediately before treatment i.e. before the intervention and immediately post treatment and the subjects were instructed to complete the pain scales again in 30 minutes, 60 minutes, 120 minutes post treatment and the next morning upon awakening. Each subject was instructed not to take any pain medication until after completion of all the pain scales unless it was absolutely necessary. A self-addressed, stamped envelope was provided for return of the completed pain scales.

\section{Interventions:}

We attempted to control for other causes for the continued decrease in pain over time by requesting that the subjects take no pain medication at least four hours before treatment and if possible until all the data are collected.

\section{Acupuncture}

Treatments were carried out by a specialist physiotherapist who was a certified acupuncturist (by Acupuncture Association of Chartered Physiotherapist, UK). The design of the acupuncture intervention, and the acupuncture points were based on expert opinion ${ }^{15}, 16$, Primary acupuncture points used in this study were Bladder 21, Bladder 29, Stomach 36, and Spleen 6. Seirin $0.2 \times 30 \mathrm{~mm}$ acupuncture needles were inserted bilaterally to a depth of $\leq 2 \mathrm{~cm}$, and the needles were retained for $30 \mathrm{~min}$. All subjects received the de qi sensation (which is the needling sensation of soreness, numbness or heaviness) following initial insertion of needles and half way through the treatment session.

\section{Acupuncture-like Transcutaneous Electrical Nerve Stimulation (ACUTENS)}

NTRON 2600 TENS units were used, and eight 1.5 -in diameter karaya electrodes were placed bilaterally on the following acupuncture points: Stomach 36, Spleen 6, and Bladder 21 and 29 (same points used for acupuncture treatment). The following TENS variables were used: 1$)$ the lowest rate possible (1 pulse/sec), 2) the highest intensity that was tolerable for the subject for 30 minutes, and 3) the lowest possible pulse duration $(40 \mathrm{msec})$. If the subject could tolerate the highest intensity stimulation, the duration of the pulse was increased to the point of tolerance. Tolerance was defined as the level at which the subjects asked the investigator to stop increasing the stimulation. The TENS unit was rechecked after 5 minutes. The treatment was given for a total of 30 minutes, and the electrodes were then removed ${ }^{11}$.

\section{The Control group}

Subjects were positioned prone on a plinth for 30 minutes with pillow under her head, abdomen, and lower legs. The subjects in the acupuncture and ACUTENS Groups were positioned in the same manner as those in the Control Group.

\section{Data analysis}

The values obtained pretreatment and post treatment from the VAS and the PRI were analyzed using a one way analysis of variance (ANOVA). An alpha level of .05 was chosen as the criterion for statistical significance in all tests. A pair-wise comparison using Bonferoni test was used to follow up statistically significant main effects over time. To better assess clinical significance, the post-treatment percentage of change in PRI and VAS scores were calculated for each subject, and the mean of these values was calculated to obtain a mean percentage PRI and VAS scores for each group.

\section{Results}

The subjects ranged in age from 14 to 25 years $(18.2 \pm 2.55$ years), with a mean age of menarche of $13.06 \pm 1.22$ years (Table 1). Thirty six subjects were treated in this study with pain scales completed pretreatment, immediately post-treatment; at 30,60,120 minutes post-treatment; and the next morning. One subject in the acupuncture group did not complete the information at $120 \mathrm{~min}$ post treatment and the next morning times because she was asleep at that time. Given our small sample size, we did not want to drop this subject from the study because she was missing the last two of her six data points. Thus, we substituted her pain scores at 60 minutes post-treatment for her missing scores at 120 minutes post-treatment and the next morning; that is, we assumed no change after 60 minutes for these subjects. We analyzed the data with and without this subject, and found the same results in each analysis.

Figures 1 and 2 shows a decline in the pretreatment mean pain values for the acupuncture and ACUTENS groups using the PRI and VAS respectively immediately post-treatment which was also maintained at 30,60, 120 minutes post-treatment; and the next morning. The ANOVA test revealed no statistical significance difference in the pretreatment scores of PRI of McGill pain questionnaire $(\mathrm{P}=0.989)$ and VAS $(\mathrm{P}=1.00)$. However, statistical significance difference $(\mathrm{P}=0.00)$, in PRI and VAS scores were obtained at all post-treatment points of data collection (Table 2). Post hoc analysis of the differences in PRI and VAS scores 
between the Acupuncture and ACUTENS groups were not statistically significant $(P>0.05)$ (Table 2). Thus, pain relief was approximately the same for the acupuncture and the ACUTENS groups over time. At the immediate post-treatment measurement time, the PRI values from McGill pain questionnaire revealed a $10.41 \%$ decrease in pain for the control Group and a 50.28\% decrease in pain for the acupuncture group and $52.26 \%$ for the ACUTENS group (Figure 3). The VAS scores recorded immediately post treatment showed $8.89 \%$ reduction in pain for the control group, $74.58 \%$ for the acupuncture group and $75.55 \%$ for the ACUTENS group.

\section{Discussion}

The outcome of the present study showed that acupuncture and acupuncture like transcutaneous electrical nerve stimulation treatment might be effective in treating pain due to primary dysmenorhea. Previous studies have reported similar findings $\mathrm{s}^{14,15,16,17}$, Steinberger used acupuncture at four acupuncture points for five consecutive days before the menstrual cycle of 48 women and $58 \%$ of the subjects had total cessation of pain before and during menstruation, and $25 \%$ had greatly decreased symptoms. The pain relief lasted up to six months post treatment ${ }^{13}$. Helms used acupuncture on 43 dysmenorrheic women, although the results of the study were not statistically significant, $91 \%$ of the subjects in the acupuncture group showed improvement with pain reduction. Improvement in Helms' study was defined as an average post-treatment score of less than half of the pretreatment pain score ${ }^{9}$. This study did reveal a significant decrease in pain over time for both the acupuncture and ACUTENS groups which lasted as long as hours and days have been documented by other researchers after treatment with TENS ${ }^{12,13,14}$

According to the concept of traditional Chinese medicine ideas, dysmenorhea could be caused by an excess type disturbance with cramp type pain which increases with stress or warmth and radiate into the legs or back $^{18}$, or rarely a deficiency type disturbance, which is characterized by dull pain, dizziness, tiredness or sensitivity to cold during menstruation that is relieved by warmth ${ }^{18}$. Acupuncture stimulation activated A- $\delta$ and $\mathrm{C}$ afferent fibers in muscle ${ }^{16,17}$ during needle stimulation of acupuncture points thus, signals are transmitted to the spinal cord, and via afferent pathways to the midbrain ${ }^{16,}{ }^{17}$. The perception of pain emerges from the resulting flow and integration of this information among specific brain areas and leads to a change in the perception of pain. The descending pain-modulatory system is a key anatomical network that underlies the ability to change pain intensity ${ }^{16}$. Acupuncture analgesia improved the noxious descending inhibitory controls and pain gate mechanism and therefore helped the patients' pain levels. The overall result was that of pain reduction ${ }^{16,17}$ presently, it is difficult to ascertain to what extent acupuncture has helped these patients, as psychological components could neither be ruled out nor measured, A preliminary paper by Gamus et $\mathrm{al}^{17}$ demonstrated that pain perception and coping strategies can be positively affected by acupuncture although it is not yet clear how although as previously suggested the limbic system, somatosensory cortex, brainstem, cerebellum can be involved. It could also be that other mechanisms underlying the therapeutic effects of acupuncture on pain treatment are yet to be identified.

Mannheimer and Whalen compared the effectiveness of conventional TENS and acupuncture-like TENS and found significant relief of primary dysmenorrhea with both forms ${ }^{14}$. Kroth used acupuncture-like TENS to treat women with primary dysmenorrhea and reported that all subjects experienced more than $50 \%$ pain reduction 180 minutes after treatment. However, the study did not use a control group ${ }^{12}$. Neighbors et al in another follow-up study treated women with acupuncture-like TENS and a placebo pill ${ }^{11}$, they found a statistically significant reduction in pain immediately post-treatment for subjects treated with acupuncture-like electrical nerve stimulations, with at least $50 \%$ relief of pain. All of these studies showed significant pain relief for the subjects receiving either acupuncture or acupuncture-like TENS. Our study was design to compare the effectiveness of acupuncture and acupuncture like transcutaneous electrical nerve stimulation in the treatment of dysmenorhea. The results from our study indicated that the acupuncture and acupuncture like TENS groups received approximately the same amount of pain relief post-treatment at every points of data collection. Acupuncture-like TENS or ACUTENS involves the use of low frequency, high intensity TENS to stimulate acupuncture points to produce 'de qi' sensation of real acupuncture. With ACUTENS, small diameter, high threshold fibres are activated. Thus, stimulation with acupuncture-like TENS recruits type III fibres causing a greater release of endorphins than with the use of conventional TENS ${ }^{18}$. The results of the present study did not show any significant difference between acupuncture and ACUTENS in the level of pain reduction post treatment.

ACUTENS has advantages and disadvantages when compared with acupuncture. Advantages include omission of certain risk of using needles such as infection. Many patients have a fear of needles and prefer TENS $^{14,18}$. Perhaps the most compelling reason for TENS is the ease of use and the fact that not as much as precision is required in placement of pads over the acupuncture points as pushing the needles through the skin and muscle fibres to produce 'de qi' sensations given the large areas covered by the pad. The ease of use makes TENS more acceptable to patients (self administered for home use) and to therapist who are not certain about the points of needle placement, depth of penetration, or angle of the needle trajectory ${ }^{12.18}$. The disadvantages of 
ACUTENS must also be considered, most TENS devices cannot achieve 'de qi' sensation even at high current intensities. This is because the pulse shapes they emit cause burning and cutting sensation from skin nerve instead of 'de qi' sensation from type III muscle afferents. Even with proper TENS devices, some patients do not like strong stimulation, unpleasant sensations and strong muscular contraction accompanying ACUTENS and hence they never achieve the benefit of stimulating type III muscle afferents 'de qi' sensations which is the main goal of treating pain with acupuncture ${ }^{18}$. It could therefore be advisable that before applying ACUTENS, the units should be tried out by the clinician to see if 'de qi' sensation could be achieved without accompanying burning sensation from the skin.

\section{Limitations}

The limitations of the trial reflect the lack of power, and whether the results can be representative of women with primary dysmenorhea. Thus, continued research into the effects of acupuncture and ACUTENS in large randomized controlled trials on primary dysmenorhea with a longer follow up period is warranted.

\section{Conclusion}

Although the results of this study did not show a statistically significant difference between acupuncture and ACUTENS groups, an average of at least $50 \%$ pain reduction was noted for both groups. For this reason, acupuncture and acupuncture-like TENS could be a possible solution for women who suffer from severe dysmenorhea and do not experience pain relief with other forms of treatment.

\section{References}

[1]. Dawood MY, "Primary dysmenorrhea: advances in pathogenesis and management," Obstetrics and Gynecology 2006, 108 (2):428441

[2]. Burnett MA, Antao V, and Black A, "Prevalence of primary dysmenorrhea in Canada," Journal of Obstetrics and Gynaecology Canada 2005, 27, (8):765-770

[3]. Hillen TIJ, Grbavac SL, Johnston PJ, Straton JAY, and Keogh JMF, "Primary dysmenorrhea in young Western Australian women: prevalence, impact, and knowledge of treatment," Journal of Adolescent Health 1999, 25: 40-45

[4]. Harel Z, "Dysmenorrhea in adolescents and young adults: etiology and management," Journal of Pediatric and Adolescent Gynecology 2006, 19: 363-371

[5]. Wong CL, Farquhar C, Roberts H, and Proctor M, "Oral contraceptive pill as treatment for primary dysmenorrhoea," Cochrane Database of Systematic Reviews, no. 2, Article ID CD002120, 2009.

[6]. Sundell G, Milsom I, and Andersch B, "Factors influencing the prevalence and severity of dysmenorrhoea in young women," British Journal of Obstetrics and Gynaecology 1990, 97: 588-594

[7]. MacPherson H, Sinclair-Lian N, and Thomas K, "Patients seeking care from acupuncture practitioners in the UK: a national survey," Complementary Therapies in Medicine 2006, 14: 20-30, 2006.

[8]. Proctor ML, Smith CA, Farquhar CM, and Stones RW, "Transcutaneous electrical nerve stimulation and acupuncture for primary dysmenorrhoea," Cochrane Database of Systematic Reviews 2002

[9]. Helms JM, “Acupuncture for the management of primary dysmenorrhea," Obstetrics and Gynecology, 1987, 69: 51-56

[10]. Yang H, Liu C-Z, Chen X et al., "Systematic review of clinical trials of acupuncture-related therapies for primary dysmenorrhea," Acta Obstetricia et Gynecologica Scandinavica 2008, 87:1114-1122

[11]. Neighbors LE, Clelland JA, Jackson JR,: Transcutaneous electrical nerve stimulation for pain relief in primary dysmenorrhea. Clinical Journal of Pain 1987, 3:17-22,

[12]. Kroth MF: The Effect of Transcutaneous Electrical Nerve Stimulation on Females Experiencing Pain from Dysmenorrhea: Preliminary Findings. Master's Thesis. Birmingham, AL, The University of Alabama at Birmingham, 1983

[13]. Steinberger A: The treatment of dysmenorrhea by acupuncture. Am J Chin Med 1981, 9:57-60

[14]. Mannheimer JS, Whalen EC: The efficacy of transcutaneous electrical nerve stimulation in dysmenorrhea. Clinical Journal of Pain $1985,1: 75-83$

[15]. Maciocia G Obstetrics and Gynaecology in Chinese Medicine 1998, Churchill Livingstone, New York, NY, USA,

[16]. Streitberger and J. Kleinhenz, "Introducing a placebo needle into acupuncture research," The Lancet, 352, (912)5364-365, 1998.

[17]. Gamus D, Meshulam-Atzmon V, Pintov S, Jacoby R,. The Effect of Acupuncture Therapy on Pain Perception and Coping Strategies: A Preliminary Report. Acupuncture Meridian Studies 2008, 1:51-53.

[18]. Stux G, Pomeranz B, Bascics of Acupuncture 1998 Fourth Edition, Springer: 278-279 
Table 1: Age and onset of menarche of participants

\begin{tabular}{|l|l|l|l|l|l|l|}
\hline & $\begin{array}{l}\text { Control } \\
(\mathbf{X}+\mathbf{S D})\end{array}$ & $\begin{array}{l}\text { Acupuncture } \\
(\mathbf{X}+\mathbf{S D})\end{array}$ & $\begin{array}{l}\text { ACUTENS } \\
(\mathbf{X}+\mathbf{S D})\end{array}$ & $\begin{array}{l}\text { Total } \\
(\mathbf{X} \pm \text { SD) }\end{array}$ & F-values & P-values \\
\hline Age (yrs) & $18.75 \pm 3.17$ & $18.67 \pm 2.15$ & $17.25 \pm 2.14$ & $\begin{array}{l}18.2 \\
\pm 2.55\end{array}$ & 1.33 & 0.278 \\
\hline $\begin{array}{l}\text { Onset of Menarche } \\
\text { (yrs) }\end{array}$ & $13.17 \pm 1.19$ & $13.17 \pm 1.14$ & $12.84 \pm 1.40$ & $\begin{array}{l}13.06 \pm \\
1.22\end{array}$ & 0.288 & 0.752 \\
\hline
\end{tabular}

Table 2: ANOVA table for PRI and VAS scores

\begin{tabular}{|c|c|c|c|c|c|c|}
\hline & & SS & df & MS & $\mathbf{F}$ & Sig. \\
\hline PRI sores pre treatment & $\begin{array}{l}\text { Between Groups } \\
\text { Within Groups }\end{array}$ & $\begin{array}{l}.222 \\
235.000\end{array}$ & $\begin{array}{l}2 \\
33\end{array}$ & $\begin{array}{l}.111 \\
7.121\end{array}$ & .016 & .985 \\
\hline PRI Scores post-treatment & $\begin{array}{l}\text { Between Groups } \\
\text { Within Groups }\end{array}$ & $\begin{array}{l}1415.389 \\
267.500\end{array}$ & $\begin{array}{l}2 \\
33\end{array}$ & $\begin{array}{l}707.694 \\
8.106\end{array}$ & 87.304 & .000 \\
\hline PRI Scores 30 mins & $\begin{array}{l}\text { Between Groups } \\
\text { Within Groups }\end{array}$ & $\begin{array}{l}1470.389 \\
229.833\end{array}$ & $\begin{array}{l}2 \\
33\end{array}$ & $\begin{array}{l}735.194 \\
6.965\end{array}$ & 105.561 & .000 \\
\hline PRI Scores 60mins & $\begin{array}{l}\text { Between Groups } \\
\text { Within Groups }\end{array}$ & $\begin{array}{l}2348.167 \\
264.583\end{array}$ & $\begin{array}{l}2 \\
33\end{array}$ & $\begin{array}{l}1174.083 \\
8.018\end{array}$ & 146.437 & .000 \\
\hline PRI Scores 120mins & $\begin{array}{l}\text { Between Groups } \\
\text { Within Groups }\end{array}$ & $\begin{array}{l}2429.389 \\
254.500\end{array}$ & $\begin{array}{l}2 \\
33\end{array}$ & $\begin{array}{l}1214.694 \\
7.712\end{array}$ & 157.505 & .000 \\
\hline PRI Scores Day 2 & $\begin{array}{l}\text { Between Groups } \\
\text { Within Groups }\end{array}$ & $\begin{array}{l}2450.000 \\
294.750\end{array}$ & 33 & $\begin{array}{l}1225.000 \\
8.932\end{array}$ & 137.150 & .000 \\
\hline VAS Scores pretreatment & $\begin{array}{l}\text { Between Groups } \\
\text { Within Groups }\end{array}$ & $\begin{array}{l}.000 \\
66.750\end{array}$ & $\begin{array}{l}2 \\
33\end{array}$ & $\begin{array}{l}.000 \\
2.023\end{array}$ & .000 & 1.000 \\
\hline VAS scores post treatment & $\begin{array}{l}\text { Between Groups } \\
\text { Within Groups }\end{array}$ & $\begin{array}{l}95.722 \\
43.167\end{array}$ & $\begin{array}{l}2 \\
33\end{array}$ & $\begin{array}{l}47.861 \\
1.308\end{array}$ & 36.589 & .000 \\
\hline VAS scores 30 minutes & $\begin{array}{l}\text { Between Groups } \\
\text { Within Groups }\end{array}$ & $\begin{array}{l}95.722 \\
43.167\end{array}$ & $\begin{array}{l}2 \\
33\end{array}$ & $\begin{array}{l}47.861 \\
1.308\end{array}$ & 36.589 & .000 \\
\hline VAS scores $60 \mathrm{mins}$ & $\begin{array}{l}\text { Between Groups } \\
\text { Within Groups }\end{array}$ & $\begin{array}{l}125.722 \\
35.167\end{array}$ & 33 & $\begin{array}{l}62.861 \\
1.066\end{array}$ & 58.988 & .000 \\
\hline VAS scores $120 \mathrm{mins}$ & $\begin{array}{l}\text { Between Groups } \\
\text { Within Groups }\end{array}$ & $\begin{array}{l}144.667 \\
30.083\end{array}$ & $\begin{array}{l}2 \\
33\end{array}$ & $\begin{array}{l}72.333 \\
.912\end{array}$ & 79.346 & .000 \\
\hline VAS scores day 2 & $\begin{array}{l}\text { Between Groups } \\
\text { Within Groups }\end{array}$ & $\begin{array}{l}137.167 \\
29.833\end{array}$ & $\begin{array}{l}2 \\
33\end{array}$ & $\begin{array}{l}68.583 \\
.904\end{array}$ & 75.863 & .000 \\
\hline
\end{tabular}

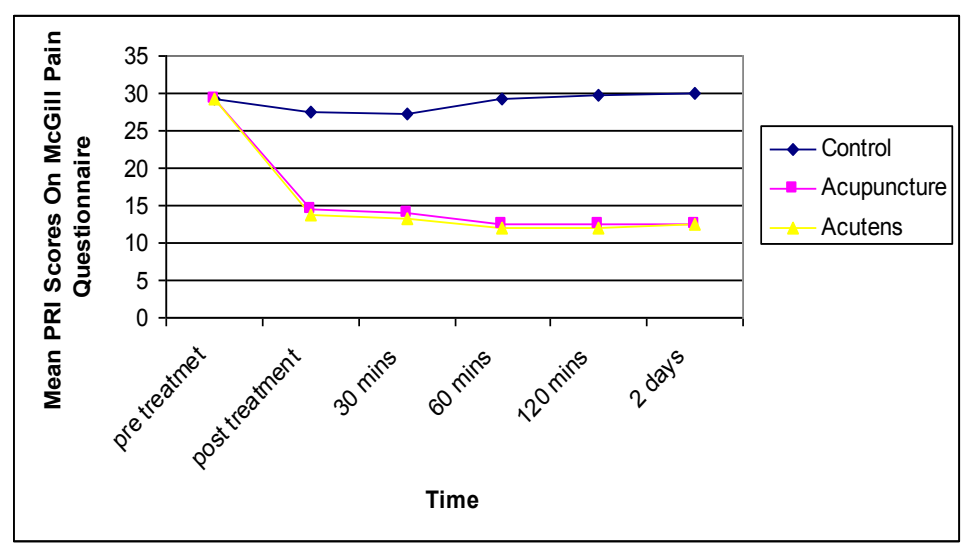

Figure 1: Mean PRI Scores on McGill pain Questionnaires 


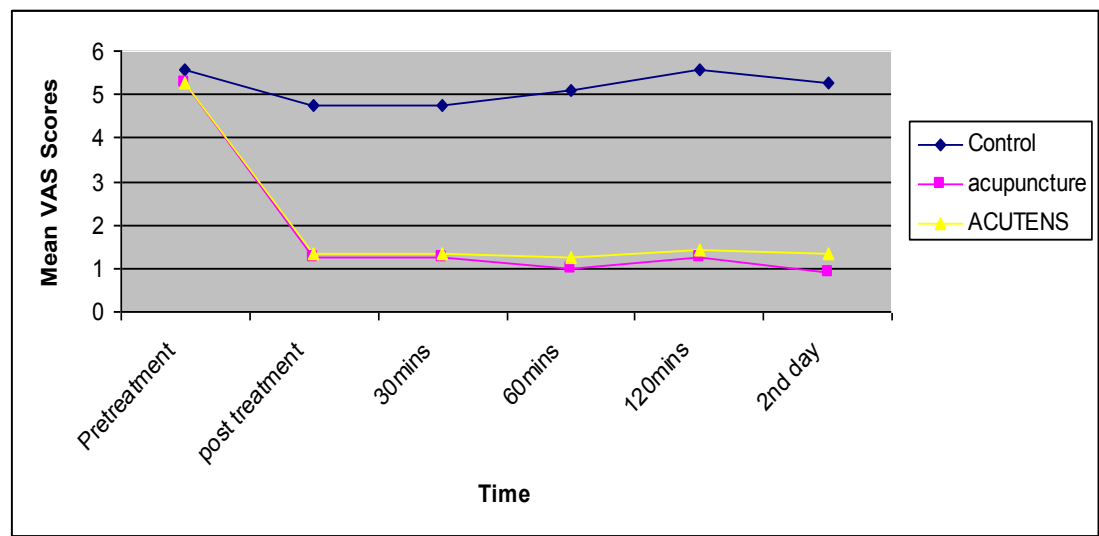

Figure 2: Mean VAS Scores

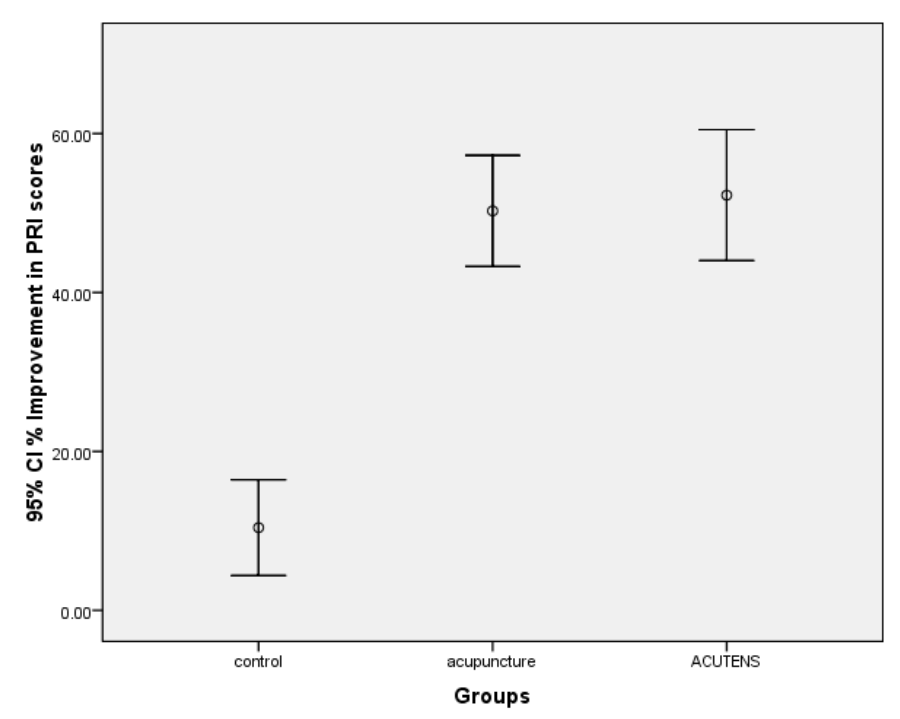

Figure 3: \% Improvement in PRI Scores

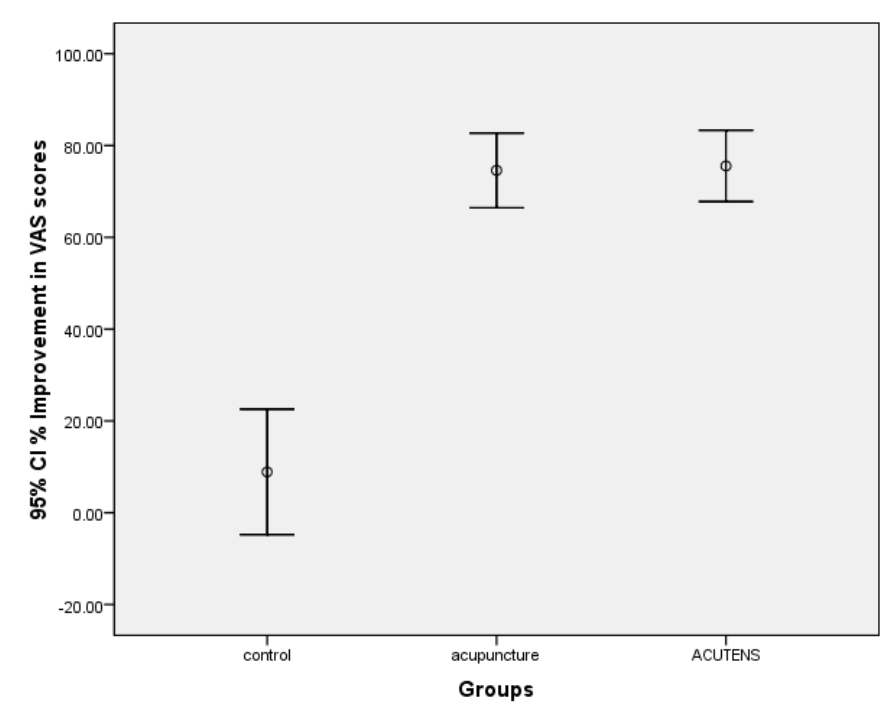

Figure 4 \% Improvement in VAS scores 